\title{
Best Practice in Intravascular Lithotripsy
}

\author{
Benjamin Honton (1' and Jacques Monsegu²
}

1. Department of Interventional Cardiology, Clinique Pasteur, Toulouse, France; 2. Department of Interventional Cardiology, Institut Cardio-Vasculaire, Groupe Hospitalier Mutualiste, Grenoble, France

\begin{abstract}
Intravascular lithotripsy (IVL) is a novel approach to lesion preparation of severely calcified plaques in coronary and peripheral vessels. Lithotripsy is delivered by vaporising fluid to create an expanding bubble that generates sonic pressure waves that interact with arterial calcification. Available data indicate that IVL leads to increased vessel compliance before stent implantation with high efficacy and an excellent safety profile. Since it gained the CE mark in 2017, and with improved operator experience, the use of IVL has expanded into more complex clinical situations. This review focuses on the best practice for IVL use in the cath lab, based on 3 years of experience with the technology and the latest scientific data from the Disrupt CAD clinical trials.
\end{abstract}

\section{Keywords}

Intravascular lithotripsy, coronary calcified lesion, best practice, plaque modification, Disrupt CAD

Disclosure: BH receives speaker fees from Shockwave. JM has no conflicts of interest to declare.

Acknowledgment: Editorial support was provided by Pelle Stolt, Basel, Switzerland.

Received: 14 May 2021 Accepted: 23 September 2021 Citation: Interventional Cardiology 2022;17:e02. DOI: https://doi.org/10.15420/icr.2021.14

Correspondence: Benjamin Honton, Clinique Pasteur, 45 avenue de Lombez, BP 27617, 31076 Toulouse, France. E: bhonton@clinique-pasteur.com

Support: The publication of this article was supported by Shockwave Medical Inc.

Open Access: This work is open access under the CC-BY-NC 4.0 License which allows users to copy, redistribute and make derivative works for non-commercial purposes, provided the original work is cited correctly.

The prevalence of calcified coronary lesions encountered in daily percutaneous coronary intervention $(\mathrm{PCl})$ is set to increase with the growing prevalence of predisposing factors such as hypertension, ageing and diabetes. ${ }^{1}$ Calcified lesions lead to sub-optimal PCl outcomes by limiting the crossing of lesions, altering drug elution kinetics and interfering with optimal stent expansion, and they are also associated with poorer clinical prognosis. ${ }^{1-6}$ Common technologies to treat calcified plaque, such as rotational or orbital atherectomy, are associated with increased periprocedural complications without clear clinical evidence of efficacy. ${ }^{7}$ There is thus an unmet need for effective and safe methods to prepare calcified lesions and improve $\mathrm{PCl}$ outcomes.

\section{Intravascular Lithotripsy: Technical and Scientific Overview}

Intravascular lithotripsy (IVL) is a new vessel preparation technique for calcified coronary artery lesions that creates multiplane micro/macro fractures in the calcified plaque before stenting or allowing for an improved stent expansion. ${ }^{8}$ The C2 Shockwave Medical Coronary IVL system (Shockwave Medical) consists of three components: a generator; a connector cable and a sterile catheter incorporating the lithotripsy emitters enclosed in a semi-compliant balloon (Figure 1). IVL emitters produce electric sparks that create vapour bubbles in the surrounding fluid medium in the integrated balloon. IVL produces low levels of electric energy, leading to the formation and rapid expansion of vapour bubbles, resulting in acoustic pressure waves that radiate circumferentially and transmurally in an unfocused manner. These acoustic pressure waves interact with high-density tissues such as calcium without affecting soft tissue. This interaction disrupts the calcium by creating micro-macro fractures, and increases vessel compliance. ${ }^{8}$

The catheter, available in $2.5-4.0 \mathrm{~mm}$ diameters, is programmed to deliver 10 pulses in sequence at a frequency of 1 pulse/second for a maximum of 80 pulses per catheter. The low pressure inflation avoids the barotraumatic vessel wall injuries related to high pressure inflation. ${ }^{8,9}$ The role of the fluid-filled integrated balloon is to facilitate efficient transmission of shockwave energy to vascular tissue by several mechanisms: creation of the spark which requires ions, adequate interface with similar acoustic impedances, avoiding thermal injury, and shielding the emitters from direct contact with the arterial wall. ${ }^{8}$ Compared with atherectomy, the IVL acoustic burst penetrates deeper into the arterial wall to generate multiplane longitudinal fractures without affecting healthy tissue.

The coronary IVL system has received both the CE Mark and FDA approval. IVL has been studied in the Disrupt CAD clinical trials, including the pivotal Disrupt CAD III study. The most recent data confirms that IVL is a safe procedure with a high success rate - primary safety and effectiveness endpoints were achieved in $92.7 \%$ and $92.4 \%$ in the pooled analysis of all Disrupt clinical trials. At 30 days, the rates of target lesion failure, cardiac death and stent thrombosis were $7.2 \%, 0.5 \%$ and $0.8 \%$, respectively. ${ }^{10}$ However, patients in such trials are highly selected and are not representative of daily clinical practice.

The aim of this review is to focus on best IVL practice based on the authors' 3 years of experience and the latest scientific data from Disrupt CAD. 
Figure 1: Intravascular Lithotripsy Shockwave System

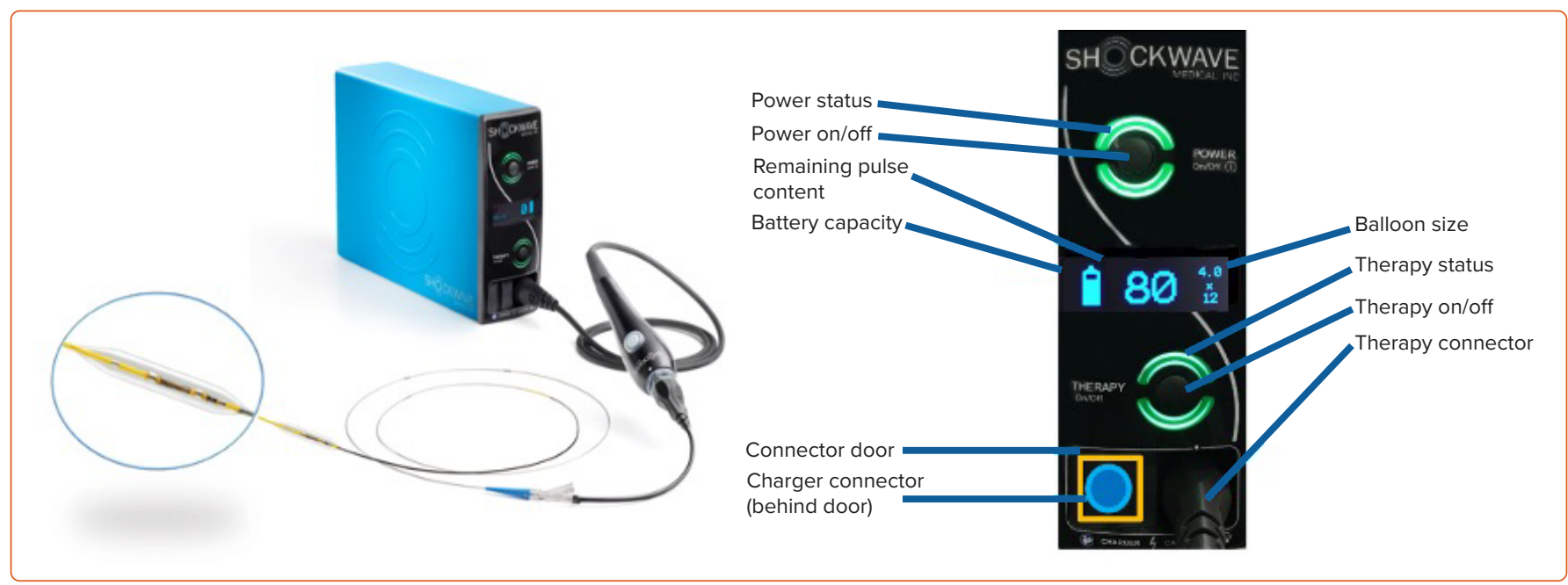

The system is composed of a generator - a connector and the C2 catheter. The generator has two buttons: the upper button switches on the generator and the lower button allows the delivering of the therapy by the generator. Note that for security reasons, you cannot plug the generator to the general electric alimentation through the charge connector when the connector is plugged to the therapy connector. The connector is related to the catheter through a magnetic plug and supports the therapy button. Reproduced with permission from Shockwave Medical.

\section{Intravascular Lithotripsy Catheter Preparation}

As the acoustic shockwaves are propagated through fluid and are impaired by air, the first step is to wash out the air in the catheter using a standard technique. This stage is essential to ensure optimal transmission of the sonic wave into intimal and medial layers. We recommend filling a syringe with $5 \mathrm{cc}$ of 50/50 saline/contrast medium and connecting the syringe to the inflation port on the catheter hub. Pull vacuum at least three times to allow the fluid to replace the air in the catheter. Disconnect the syringe and connect the inflator device with $10 \mathrm{cc}$ of 50/50 saline/contrast medium to the inflation port, ensuring no air is introduced into the system.

Supplementary Material Figure 1 shows a step-by-step guide to therapy delivery.

\section{Intravascular Lithotripsy Target Lesion Selection: Concentric or Eccentric Calcification?}

As the IVL emitters generate a circumferential acoustic wave, arterial circumferential calcification is the most suitable target for IVL. Moreover, part of the acoustic wave is transferred across the calcified plaque and reflected, interacting with the opposite side of the lesion in a process known as spallation, which increases the procedure's efficiency." Circumferential calcification modification increases vessel compliance and allows for full symmetrical stent expansion. For these reasons, circumferential calcification was an inclusion criterion in the Disrupt CAD trials, defined by the presence of fluoroscopic radio-opacities without cardiac motion involving both sides of the arterial wall, or by the presence of $\geq 270$ degrees of calcium on at least one cross-section on intravascular imaging. ${ }^{12-14}$ Other criteria were a diameter stenosis $\geq 70 \%$, native coronary artery lesion length $\leq 40 \mathrm{~mm}$ and heavy calcification, defined as calcification within the lesion on both sides of the vessel assessed during angiography. ${ }^{14}$

There is evidence that IVL may also be appropriate for eccentric lesions as suggested by a post-hoc analysis in a pooled patient population with eccentric calcified lesions (identified by an independent core lab) in Disrupt CAD I and II..$^{15}$ Eccentric lesions were defined as a stenotic lesion that had one of its luminal edges in the outer one-quarter of the apparently normal vessel lumen whereas concentric lesion has the same criteria but involving both luminal edges.
We found a high procedural success rates, defined as a residual stenosis of $<50 \%$ after stenting without intra-hospital major adverse cardiovascular events (MACE) of $93.6 \%$ and $93.2 \%$ in eccentric and concentric lesions, respectively. There was no difference in vascular complications and clinical outcomes according to the definitions of coronary artery disease (CAD). No difference in mean target delivery pulses between the two groups have been noted for reaching procedural success. However, over 3 years of IVL use in clinical practice, several users have suggested that more pulses may be required to modify calcium in eccentric lesions due to the emitter being at a greater distance from the plaque and the lack of wave reflection compared with concentric lesions. From our experience, we recommend using a 1.1 non-compliant (NC) balloon post IVL therapy to determine the effectiveness of the IVL procedure and to assess the need for an additional IVL catheter.

\section{Intravascular Lithotripsy Therapy Application}

The catheter diameter should be selected at a 1:1 ratio relative to the target-vessel diameter and inflated at a sub-nominal pressure (4 atm). Proper apposition of the catheter to the arterial wall is necessary for an adequate fluid/tissue interface to optimise acoustic energy transfer. As noted above, there are several mechanisms responsible for the disruption; in addition to spallation squeezing, cavitation and plaque fatigue that all play a role, as detailed in a recent review. ${ }^{11}$ The estimated peak pressure of the wave is $50 \mathrm{~atm}$. Notably, the wave and not the balloon generates the disruptive force. This has several advantages - it allows low-pressure balloon inflation which reduces the risk of barotrauma, vascular dissection and perforation and post-dilation after IVL for residual stenosis before stenting (defined in Disrupt CAD III as a residual stenosis $>50 \%$ ) is often unnecessary. In the Disrupt CAD III study, post-dilation was used in only $20.7 \%$ of cases. ${ }^{14}$

In clinical practice, most operators recommend performing an NC balloon after dilation only if there is an incomplete expansion of IVL catheters of more than $30 \%$. It remains unclear how many pulses need to be delivered to ensure good lesion preparation. One catheter can deliver a maximum of 80 pulses.

On average, two IVL catheters were used in the Disrupt CAD I and 1.2 were employed in Disrupt CAD II and III. In the Disrupt pooled analysis, 
Figure 2: Decision Algorithm for Treatment of Calcified Coronary Vessels

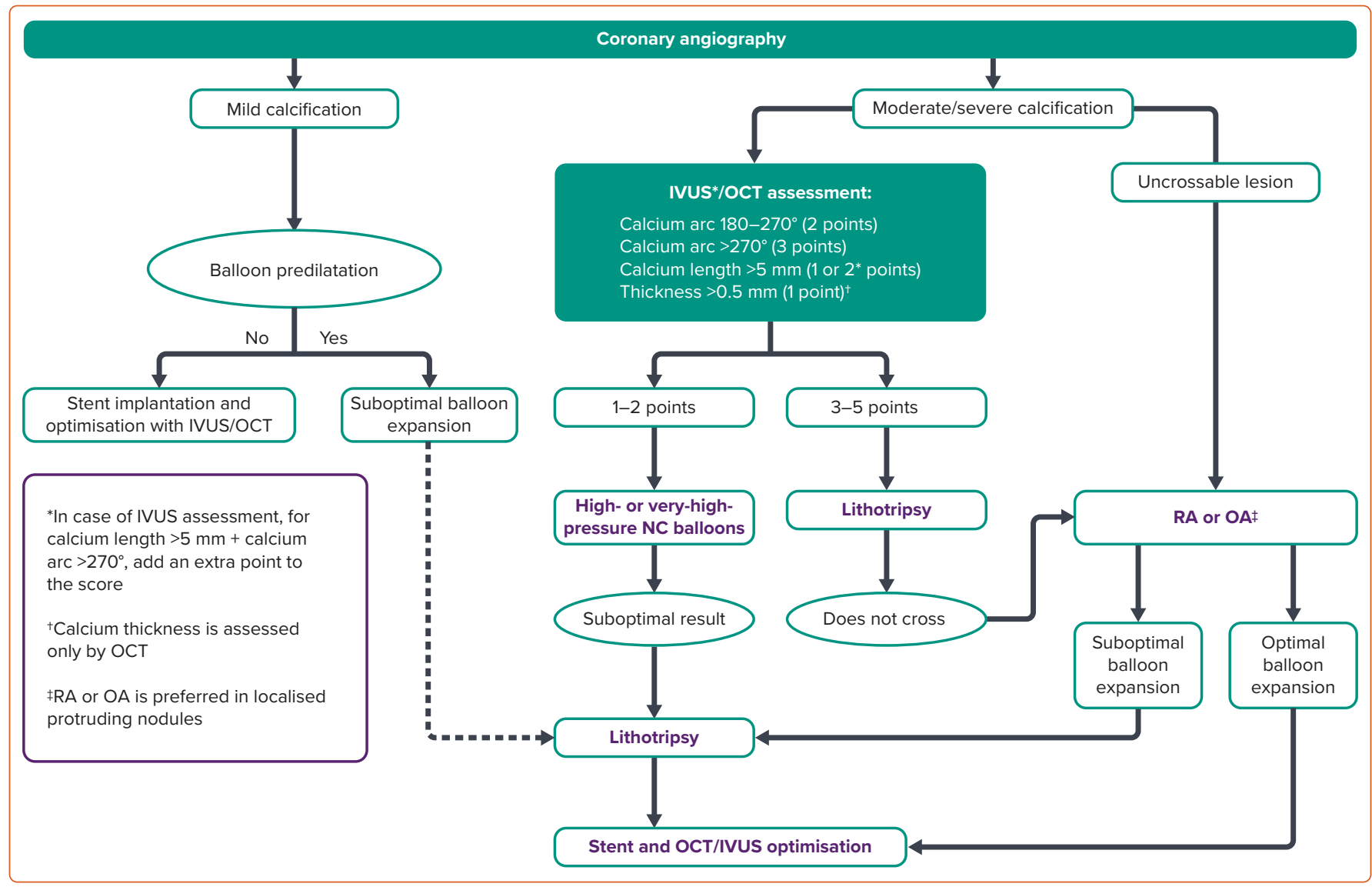

IVUS = intravascular ultrasound; NC = non-compliant; $O A$ = orbital atherectomy; OCT = optical coherence tomography; $R A=$ rotational atherectomy. Source: Sorini Dini et al. 2019. ${ }^{19}$ Reproduced with permission from Radcliffe Cardiology.

the mean number of pulses were $74.7 \pm 42.7$ and $1.3 \pm 0.6$ catheter was used..$^{10}$ According to protocol in Disrupt CAD I, II and III, we assume that a minimum of 20 pulses delivered to the target lesion should be the lowest threshold required for pulse delivery if there is no residual footprint on the balloon.

\section{Use of Endovascular Imaging}

Endovascular imaging may be useful for pre- and post-procedural evaluations.

\section{Pre-procedural Evaluation}

Coronary angiography often underestimates calcium. ${ }^{16}$ In our experience, optical coherence tomography (OCT) is the preferred imaging modality due to its high spatial resolution and ability to measure calcification thickness. Intravascular ultrasound imaging (IVUS) is a valid alternative. In an in vivo sensitivity assessment of 440 lesions, OCT detected calcium in $76.8 \%$ and IVUS in $82.7 \%$ of lesions, whereas angiography identified calcium in only $40.2 \%$ of lesions. ${ }^{17}$

Several studies have suggested a pre-defined algorithm for analysing plaque characteristics and guiding the selection of $\mathrm{PCl}$ strategies and plaque preparation using OCT or IVUS (Figure 2) ${ }^{18,19}$ We consider IVL the therapy of choice in highly calcified lesions defined as having a severe calcium $\operatorname{arc}\left(>270^{\circ}\right)$ or calcium thickness $>0.5 \mathrm{~mm}$.

Moreover, an interesting finding from the DISRUPT CAD I OCT sub-study is that more extensive calcium modifications, defined by incidence of calcium fracture, calcium fracture per lesion and quadrants of calcium
Figure 3: Typical Multiplane and Longitudinal Optical Coherence Tomography Fractures with Immediate Lumen Gain Increased After Stent Delivery

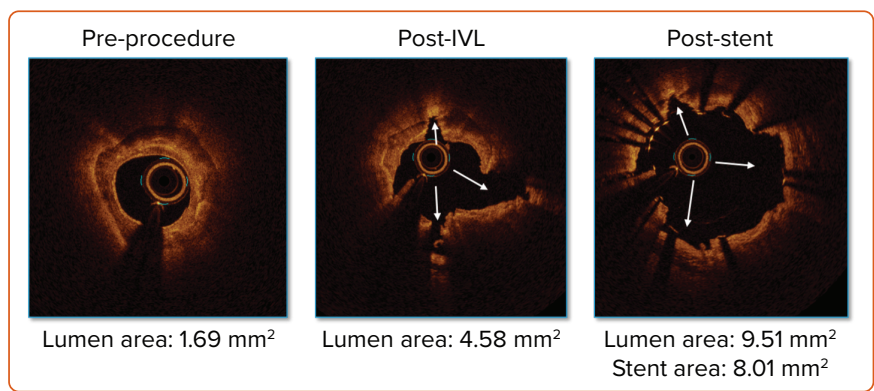

IVL = intravascular lithotripsy.

fracture, were achieved in lesions in the highest calcification tertile, suggesting that the higher the degree of calcification, the greater the IVL efficacy. ${ }^{20}$

\section{Post-therapeutic Evaluation}

Multiplane and longitudinal fractures are typically observed on OCT after IVL therapy (Figure 3). In a sub-study core lab OCT analysis in DISRUPT CAD III, these fractures resulted in increased vessel compliance with a luminal gain of $1.41 \mathrm{~mm}^{2}$ and $4.35 \mathrm{~mm}^{2}$ at the site of the minimal lumen area after IVL and stent delivery, a mean post-procedural minimal stent area of $6.66 \mathrm{~mm}^{2}$ and full stent expansion defined as mean stent expansion at the maximal calcium site. ${ }^{14}$ The percentage of lesions with calcium fractures and the maximum calcium fracture depth were similar 
Figure 4: Calcic Microfractures Induced by Intravascular Lithotripsy in Micro CT and Histology

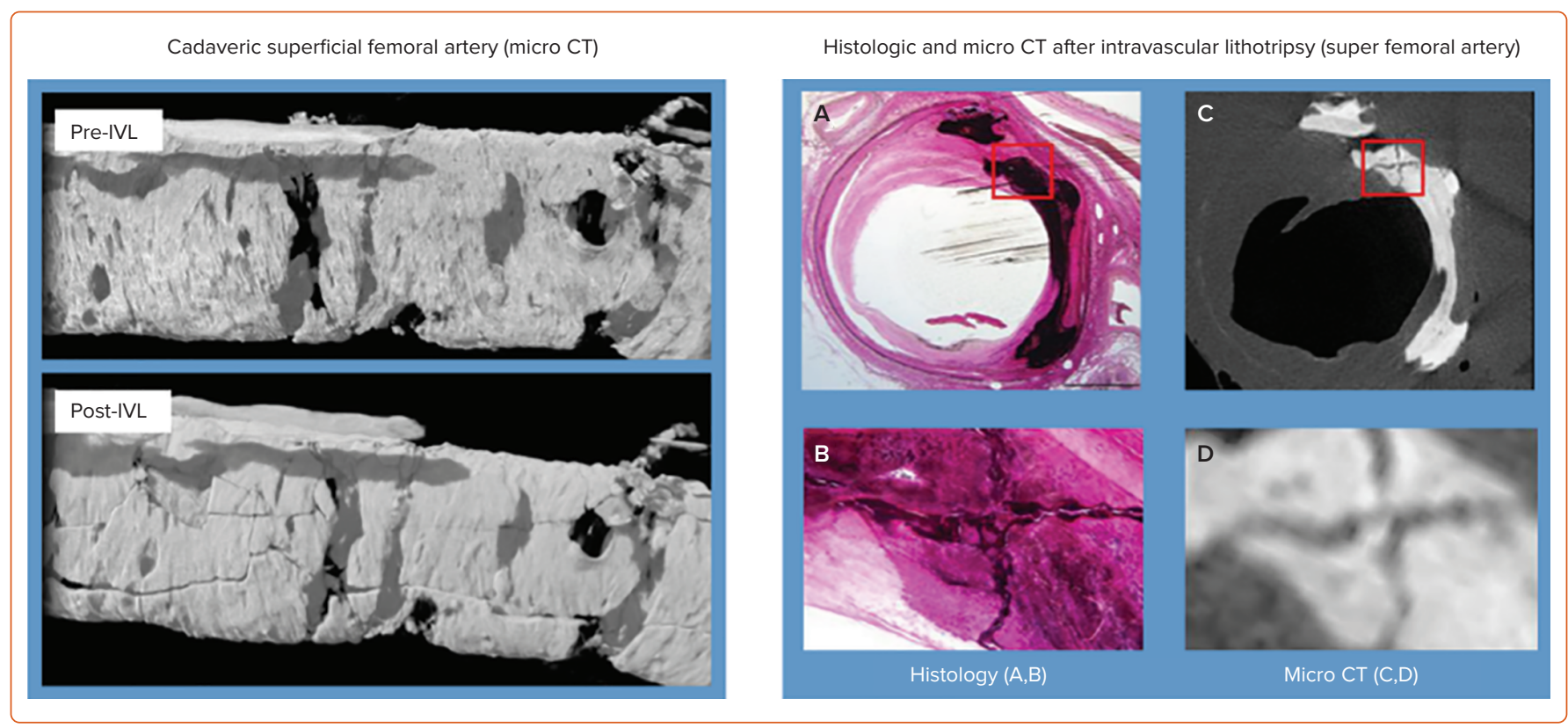

Left panel: Representative micro CT images before and after intravascular lithotripsy (IVL) treatment. Abluminal view of the left distal femoral artery demonstrating predominantly medial calcification. (A) Before (A) and after (B) IVL. Circumferential, transverse, and longitudinal calcium fractures were observed following IVL treatment. Right panel: Histological and micro CT imaging after IVL treatment. Cross-sectional histological Exakt ground section (A) matched with the micro CT cross-sectional image (C). Both sections show cross-shaped cracks highlighted by red boxed areas, which are shown at high-power magnification (B,D). Source: Kereiakes et al. 2021. ${ }^{8}$ Adapted with permission from Elsevier.

Figure 5: Intravascular Lithotripsy in Severe Calcified Unprotected Left Main Lesion
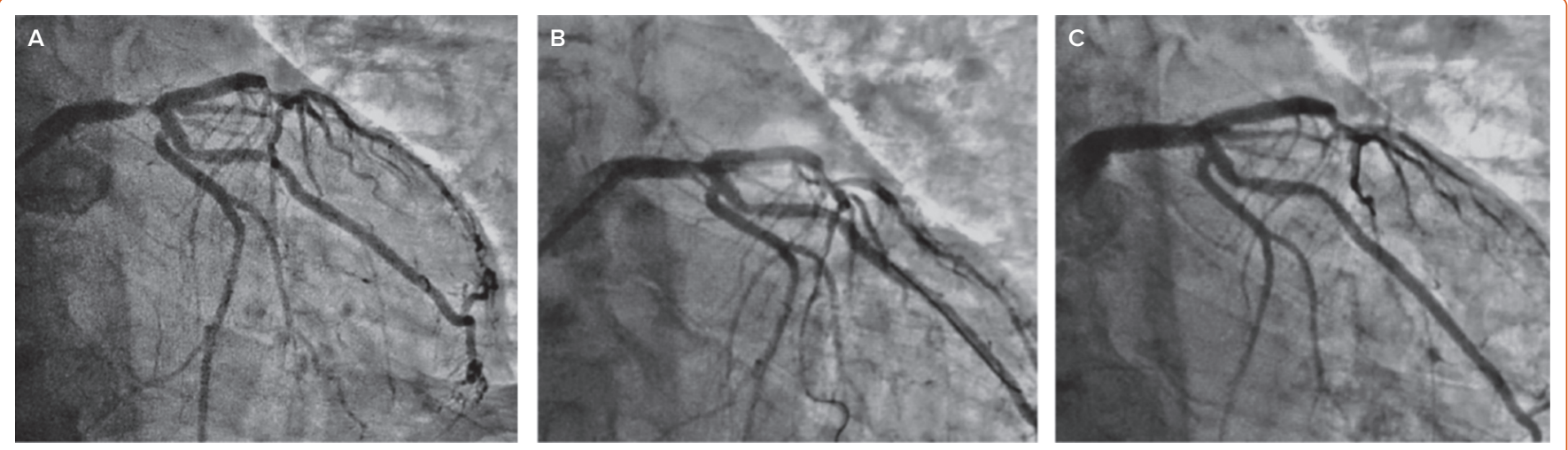

A: Severe calcified unprotected LM lesion (Medina 1-1-0); B: After application of 40 pulses $-4 \times 12 \mathrm{~mm}$ C2 catheter; C: After stent implantation, T-provisional strategy $4 \times 22 \mathrm{~mm}$ and $5 \mathrm{~mm}$ proximal optimisation technique.

in post-IVL and post-stent acquisition; however, the maximum fracture width increased after stent expansion (from $0.55 \pm 0.45 \mathrm{~mm}$ after IVL to $1.32 \pm 1.04 \mathrm{~mm}$ after stent implantation). Another notable finding was that fracture incidence occurred in $67.7 \%$ of patients but without differences in angiographic, OCT or clinical outcomes. ${ }^{14}$ This suggests that the absence of calcium fracture on OCT is not a sign of failed therapy as the fracture can be 'out of plane' and acoustic waves induce calcification microfractures beyond the resolution of OCT technology, as shown on micro-CT and histology in Figure $4 .^{8}$

\section{Limitation of Endovascular Imaging}

Access to intravascular imaging can be limited in different countries and can be because of cost. Moreover, crossability of the probes (IVUS or OCT) through complex calcified lesions can be challenging. Careful analysis of the baseline angiogram before contrast injection is crucial to classify the lesion according to the classification used by Mintz et al. and to predict $\mathrm{PCl}$ complexity and IVL success. ${ }^{21}$

\section{Specific Clinical Situations}

\section{Left Main Lesions}

The feasibility and safety of IVL in calcified left main (LM) lesions are supported by a retrospective analysis of 31 lesions treated by IVL. In this study, the target minimal stent area was achieved in $97.3 \%$ of stented segments with no in-hospital MACE. ${ }^{22}$

Similarly, good results were provided in a prospective analysis of a registry cohort of 23 patients, in which the primary endpoint (successful stent delivery and expansion with attainment of $<30 \%$ in-stent residual stenosis of the target lesion in the presence of thrombolysis in $\mathrm{Ml}$ flow grade 3) was achieved in all patients. ${ }^{23}$ In this study, the mean IVL catheter diameter used was $3.7 \pm 0.3 \mathrm{~mm}$, and a median of eight cycles and 80 pulses were applied (interquartile range: $47-80$ ). In large LM lesions, a $4.0 \mathrm{~mm}$ IVL catheter may be used to fracture the calcium, followed by a 1:1 sized NC balloon to expand the fractures created by IVL and prepare for a larger stent (Figure 5). 
Among the concerns with IVL for LM lesions is the need for prolonged vessel occlusion to deliver the required energy, which could lead to severe ischaemia.

Salazar et al. distributed the energy by providing pulses individually or in small groups with shorter balloon inflations to minimise this risk. With this strategy, only 2 of 23 patients experienced severe arterial hypotension during inflation. ${ }^{23}$

\section{Nodular Calcium Lesions}

In these lesions, more pulses may be required to modify eccentric calcium as the emitter is further away from the calcium root, and there is none of the wave reflection seen with concentric calcium. If all 80 pulses have been applied, an $\mathrm{NC}$ balloon can help determine the procedural efficacy and whether an additional IVL catheter is needed to ensure full balloon expansion.

\section{Long and Multiple Lesions}

Ideally, IVL treatment should be applied from distal to proximal lesions. However, in long lesions, the operator can start by advancing the catheter as distally as possible, delivering energy and continuing to advance the catheter. To ensure full therapeutic coverage in the vessel, a $2 \mathrm{~mm}$ overlap is advisable when delivering energy in consecutive treatment zones. In case of multiple lesions, we recommend the delivery of 20 pulses for each lesion and subsequently assessing the optimal points for remaining pulses. It needs to be kept in mind that multiple wires within the catheter enable transmission of the energy from the generator to the emitters, which may be damaged during rigorous manipulation.

\section{Difficulty in Crossing the Lesion with IVL}

If the electrodes embedded in the balloon are a prowess of technical miniaturisation, the catheter has a low profile, with a bench crossing profile of $0.042 " \pm 0.002$. Every tip and trick used in advanced $\mathrm{PCl}$, such as buddy wire, anchoring and guiding catheter extension, can be applied to overcome deliverability issues. The $6 \mathrm{Fr}$ guiding catheter extensions are compatible with all the diameters of the $\mathrm{C} 2$ catheter.

\section{Critical Lesions}

In Disrupt CAD II, pre-dilation with a balloon catheter was allowed to ensure crossing of the IVL catheter. This was necessary in $41.7 \%$ of cases, using an average balloon size of $2.2 \pm 0.6 \mathrm{~mm} .{ }^{11}$ In case of failure to cross, a hybrid rotational atherectomy approach with additional intracoronary lithotripsy (rotatripsy) can be used (as previously described by JuradoRomàn et al.) to deliver IVL and modify both superficial and deep calcium, especially in large vessels. ${ }^{24}$

From a technical perspective, it is essential to have sufficient space in the balloon to allow the formation of vapour bubbles to optimise sonic output. However, as the combination of atherectomy and IVL increased the cost of the procedure, this hybrid approach should be reserved in case of failure of one device (IVL or atherectomy bail out) and not as a front-line technique. The analysis of the pre-PCl angiogram is crucial to decide which are the best tools according to local resources and operator experience.

\section{IVL and Stent Underexpansion}

Stent underexpansion is a dramatic situation in coronary intervention leading to in-stent restenosis and/or stent thrombosis. ${ }^{25}$ Although there are numerous clinical cases in the literature reporting the efficacy of IVL in the treatment of restenosis related to underexpanded coronary stent, there are no robust data to support this off-label indication for IVL. ${ }^{26}$ A study with a smaller cohort by Aksoy and al. suggests that using IVL in an in-stent restenosis (ISR) cohort has a procedural success lower than for de novo lesions. ${ }^{27}$ Moreover, there are still pending questions regarding the necessity for using an antiproliferative drug after IVL or potential mechanical consequences of the acoustic burst on metallic scaffolding. However, stent underexpansion related to inadequate plaque preparation is a dramatic situation in which IVL could be a game-changer. More clinical data from daily practice and large-scale registry are needed.

\section{Shocktopics and Electrophysiological Disorders}

IVL generates mechanical pulses, which may cause atrial or ventricular capture in patients with bradycardia. In patients with implantable pacemakers and defibrillators, the asynchronous capture may interact with the sensing capabilities. It is essential to understand that no electrical current leaves the IVL catheter. Instead, a small amount of mechanical energy is transferred to the vessel wall when sonic pressure waves are created that have been shown to create a stretch-activated response in the myocardium. In the event of clinically significant haemodynamic effects, you may have to temporarily interrupt the IVL therapy.

Wilson et al. first described IVL-induced ventricular capture, called 'shocktopics'. In this retrospective analysis, $77.8 \%$ of patients underwent IVL-induced ventricular capture with no resulting adverse clinical events. ${ }^{28}$ The occurrence and significance of shocktopics have been evaluated in Disrupt CAD III. ${ }^{14}$ IVL-induced capture was noted during IVL in $41.1 \%$ of cases. Decreased systolic blood pressure during the IVL procedure was more frequent in patients with IVL-induced capture than those without ( $40.5 \%$ versus $24.5 \%, p=0.0007$ ). However, the magnitude of the drop in systolic blood pressure was similar between the two groups ( $p=0.07$ ). IVL-induced capture did not result in sustained ventricular arrhythmias during or immediately after the IVL procedure in any patient and was not associated with adverse events. Multivariable Cox regression analysis identified that a heart rate of $\leq 60 \mathrm{BPM}$, male sex, and the total number of IVL pulses delivered were independent predictors of IVL-induced capture. ${ }^{14}$

However, one case of VF has been related to off-label use of IVL for instent restenosis in a right dominant coronary artery (RCA). ${ }^{29}$ The authors described a ventricular arrhythmia by IVL ventricular ectopy on a T wave. It is reasonable to think that this is the consequence of multiple parameters, including favourable electrophysiological susceptibility, pre-existing ventricular ectopy and ischaemia on dominant RCA being more prone to ventricular arrhythmia. However, it also provides a warning and highlights the need to be aware of such exceptional but potential side-effects.

\section{IVL Versus Other Calcium Modification Technologies}

Calcified plaque preparation is crucial for $\mathrm{PCl}$ success. Induced-calcium modifications are significantly different from pre-existing technology (NC balloon, high-pressure balloon, modified balloon and atherectomy device) with subsequent advantages. As described above, the efficiency is powered by acoustic burst and not balloon inflation, whereas in NC balloons or modified balloons avoids vessel wall barotraumatic injury and decreases the risk of arterial dissection. IVL does not suffer from wire bias as does atherectomy (and subsequent eccentric plaque guttering) and there is a decreased risk of vascular bed overload related to debris embolisation. IVL technology also affects the deeper calcium, whereas debulking technologies are limited to the superficial calcium, which may negatively affect vessel compliance. Last, in contrast to procedures such as atherectomy, the technology can be easily adopted, not least since 
it requires no specific training as the device is delivered similarly to standard catheter-based $\mathrm{PCl}^{30}$

\section{Conclusion}

As a novel technology, published data on IVL includes highly selected clinical situations and patients, but are reinforced by operator experience in daily clinical practice. This growing experience and new trials would fill the gaps remaining in the current scientific literature for situations not encountered in the Disrupt studies. It would be interesting to have a comparator to IVL for plaque preparation, such as a modified balloon or atherectomy. In addition, the registry databases with real-world data will continue to grow and provide valuable information, particularly on the safety of IVL. With increasing uptake, we can expect further methodological advances and information from the application of IVL to more complex clinical situations.

IVL is a promising therapy for complex calcified lesions with a short learning curve and a favourable safety profile. However, knowledge of the technical characteristics of the catheter and appropriate considerations in terms of preparation, use and specific conditions for IVL will improve daily results and outcomes in patients presenting with complex calcified coronary disease. $\square$
1. Lee MS, Shah N. The impact and pathophysiologic consequences of coronary artery calcium deposition in percutaneous coronary interventions. J Invasive Cardiol 2016;28:160-7. PMID: 26301561.

2. Wiemer M, Butz T, Schmidt W, et al. Scanning electron microscopic analysis of different drug eluting stents after failed implantation: from nearly undamaged to major damaged polymers. Catheter Cardiovasc Interv 2010;75:90511. https://doi.org/10.1002/ccd.22347; PMID: 20088011

3. Tzafriri AR, Garcia-Polite F, Zani B, et al. Calcified plaque modification alters local drug delivery in the treatment of peripheral atherosclerosis. J Control Release 2017;264:20310. https://doi.org/10.1016/j.jconrel.2017.08.037; PMID: 28867375.

4. Mori S, Yasuda S, Kataoka Y, et al. Significant association of coronary artery calcification in stent delivery route with restenosis after sirolimus-eluting stent implantation. Circ $J$ 2009;73:1856-63. https://doi.org/10.1253/circj.CJ-09-0080 PMID: 19713655

5. Kobayashi Y, Okura H, Kume T, et al. Impact of target lesion coronary calcification on stent expansion. Circ $J$ 2014;78:2209-14. https://doi.org/10.1253/circj.CJ-14-0108; PMID: 25017740.

6. Généreux P, Madhavan MV, Mintz GS, et al. Ischemic outcomes after coronary intervention of calcified vessels in acute coronary syndromes. Pooled analysis from the HORIZONS-AMI (Harmonizing Outcomes With Revascularization and Stents in Acute Myocardial Infarction) and ACUITY (Acute Catheterization and Urgent Intervention Triage Strategy) trials. J Am Coll Cardio/ 2014;63:1845-54. https://doi.org/10.1016/j.jacc.2014.01.034; PMID: 24561145

7. Abdel-Wahab M, Richardt G, Joachim Büttner H, et al. Highspeed rotational atherectomy before paclitaxel-eluting stent implantation in complex calcified coronary lesions: the randomized ROTAXUS (Rotational Atherectomy Prior to Taxus Stent Treatment for Complex Native Coronary Artery Disease) trial. JACC Cardiovasc Interv 2013;6:10-9. https://doi. org/10.1016/j.jcin.2012.07.017; PMID: 23266232.

8. Kereiakes DJ, Virmani R, Hokama JY, et al. Principles of intravascular lithotripsy for calcific plaque modification. J Am Coll Cardiol Interv 2021;14:1275-92. https://doi.org/10.1016/j. jcin.2021.03.036; PMID: 34167671.

9. Serruys PW, Katagiri Y, Onuma Y. Shaking and breaking calcified plaque: lithoplasty, a breakthrough in interventiona armamentarium? JACC Cardiovasc Imaging 2017;10:907-11. https://doi.org/10.1016/j.jcmg.2017.05.011; PMID: 28797413

10. Kereiakes DJ, Di Mario C, Riley RF, et al. Intravascular lithotripsy for treatment of calcified coronary lesions: patient-level pooled analysis of the Disrupt CAD studies. JACC Cardiovasc Interv 2021:14:1337-48, https://doi. org/10.1016/j.jcin.2021.04.015; PMID: 33939604.

11. Karimi Galougahi K, Patel S, Shlofmitz RA, et al. Calcific plaque modification by acoustic shock waves: intravascular lithotripsy in coronary interventions. Circ Cardiovasc Intery 2021;14:e009354. https://doi.org/10.1161/

CIRCINTERVENTIONS.120.009354; PMID: 32907343

12. Brinton TJ, Ali ZA, Hill JM, et al. Feasibility of Shockwave coronary intravascular lithotripsy for the treatment of calcified coronary stenoses. Circulation 2019;139:834-6. https://doi.org/10.1161/CIRCULATIONAHA.118.036531; PMID: 30715944

13. Ali ZA, Nef H, Escaned J, et al. Safety and effectiveness of coronary intravascular lithotripsy for treatment of severely calcified coronary stenoses: the Disrupt CAD II study. Circ Cardiovasc Interv 2019;12:e008434. https://doi.org/10.1161/ CIRCINTERVENTIONS.119.008434; PMID: 31553205

14. Hill JM, Kereiakes DJ, Shlofmitz RA, et al. Intravascular lithotripsy for treatment of severely calcified coronary artery disease. J Am Coll Cardiol 2020;76:2635-46. https://doi, org/10.1016/j.jacc.2020.09.603; PMID: 33069849

15. Blachutzik F, Honton B, Escaned J, et al. Safety and effectiveness of coronary intravascular lithotripsy in eccentric calcified coronary lesions: a patient-level pooled analysis from the Disrupt CAD I and CAD II studies. Clin Res Cardiol 2021;110:228-36. https://doi.org/10.1007/s00392020-01737-3; PMID: 32948882

16. Wang X, Matsumura M, Mintz GS, et al. In vivo calcium detection by comparing optical coherence tomography, intravascular ultrasound, and angiography. JACC CardiovasC Imaging 2017;10:869-79. https://doi.org/10.1016/j. jcmg.2017.05.014; PMID: 28797408

17. Mintz GS. Intravascular imaging of coronary calcification and its clinical implications. JACC Cardiovasc Imaging 2015;8:46171. https://doi.org/10.1016/j.jcmg.2015.02.003; PMID: 25882575

18. Shimamura K, Guagliumi G. Optical coherence tomography for online guidance of complex coronary interventions. Circ 2016;80:2063-72. https://doi.org/10.1253/circj.CJ-16-0846; PMID: 27616595

19. Sorini Dini C, Nardi G, Ristalli F, et al. Contemporary approach to heavily calcified coronary lesions. Interv Cardiol 2019:14:154-63. https://doi.org/10.15420/icr.2019.19.R1; PMID: 31867062

20. Ali ZA, Brinton TJ, Hill JM, et al. Optical coherence tomography characterization of coronary lithoplasty for treatment of calcified lesions: first description. JACC
Cardiovasc Imaging 2017;10:897-906. https://doi.org/10.1016/j. jcmg.2017.05.012: PMID: 28797412

21. Mintz GS, Popma JJ, Pichard AD, et al. Patterns of calcification in coronary artery disease. A statistical analysis of intravascular ultrasound and coronary angiography in 1,155 lesions. Circulation 1995;91:1959-65. https://doi. org/10.1161/01.CIR.91.7.1959; PMID: 7895353.

22. Cosgrove CS, Wilson SJ, Bogle R, et al. Intravascular lithotripsy for lesion preparation in patients with calcific distal left main disease. Eurolntervention 2020;16:76-9. https://doi.org/10.4244/EIJ-D-19-01052; PMID: 32224480.

23. Salazar CH, Gonzalo N, Aksoy A, et al. Feasibility, safety, and efficacy of intravascular lithotripsy in severely calcified left main coronary stenosis. JACC Cardiovasc Interv 2020;13:1727-9. https://doi.org/10.1016/j.jcin.2020.04.022; PMID: 32703602

24. Jurado-Román A, Gonzálvez A, Galeote G, et al. RotaTripsy: combination of rotational atherectomy and intravascular lithotripsy for the treatment of severely calcified lesions. JACC Cardiovasc Interv 2019;12:e127-9. https://doi. org/10.1016/j.jcin.2019.03.036; PMID: 31326422

25. Souteyrand G, Amabile N, Mangin L, et al. Mechanisms of stent thrombosis analysed by optical coherence tomography: insights from the national PESTO French registry. Eur Heart J 2016;37:1208-16. https://doi.org/10.1093/ eurheartj/ehv711; PMID: 26757787.

26. Tovar Forero MN, Wilschut J, van Mieghem NM, Daemen J. Coronary lithoplasty: a novel treatment for stent underexpansion. Eur Heart J 2019;40:221. https://doi. org/10.1093/eurhearti/ehy593; PMID: 30289452.

27. Aksoy A, Salazar C, Becher MU, et al. Intravascular lithotripsy in calcified coronary lesions: a prospective, observational, multicenter registry. Circ Cardiovasc Interv 2019:12:e008154. https://doi.org/10.1161/ CIRCINTERVENTIONS.119.008154; PMID: 31707803

28. Wilson SJ, Spratt JC, Hill J, et al. Incidence of 'shocktopics' and asynchronous cardiac pacing in patients undergoing coronary intravascular lithotripsy. Eurolntervention 2020;15:1429-35. https://doi.org/10.4244/EIJ-D-19-00484; PMID: 31130523

29. McGarvey M, Kumar S, Violaris A, et al. Ventricular fibrillation induced by a lithotripsy-pulse on T during coronary intravascular Shockwave lithotripsy. Eur Heart J Case Rep 2020;4:1-3. https://doi.org/10.1093/ehjcr/ytaa416; PMID: 33629000.

30. Kimball BP, Bui S, Cohen EA, et al. Early experience with directional coronary atherectomy: documentation of the learning curve. Can J Cardiol 1993:9:177-85. PMID: 8490789. 